\title{
Natural Boron and ${ }^{10} \mathrm{~B}$-Enriched Hexagonal Boron Nitride for High- Sensitivity Self-Biased Metal-Semiconductor-Metal Neutron Detectors
}

\author{
Adama Mballo, Ali Ahaitouf, Suresh Sundaram, Ashutosh Srivastava, Vishnu Ottapilakkal, Rajat Gujrati, \\ Phuong Vuong, Soufiane Karrakchou, Mritunjay Kumar, Xiaohang Li, Yacine Halfaya, Simon Gautier, \\ Paul L. Voss, Jean Paul Salvestrini, and Abdallah Ougazzaden*
}

Cite This: ACS Omega 2022, 7, 804-809

Read Online

ACCESS | Lill Metrics \& More | 回 Article Recommendations ｜ sl Supporting Information

ABSTRACT: Metal-semiconductor-metal (MSM) detectors based on $\mathrm{Ti} / \mathrm{Au}$ and Ni/ $\mathrm{Au}$ interdigitated structures were fabricated using 2.5 micrometer thick hexagonal boron nitride (h-BN) layer with both natural and ${ }^{10} \mathrm{~B}$-enriched boron. Current-voltage $(I-V)$ and current-time $(I-t)$ curves of the fabricated detectors were recorded with $\left(I_{\mathrm{N}}\right)$ and without $\left(I_{\mathrm{d}}\right)$ neutron irradiation, allowing the determination of their sensitivity $\left(S=\left(I_{\mathrm{N}}-\right.\right.$ $\left.\left.I_{\mathrm{d}}\right) / I_{\mathrm{d}}=\Delta I / I_{\mathrm{d}}\right)$. Natural and ${ }^{10} \mathrm{~B}$-enriched h-BN detectors exhibited high neutron sensitivities of 233 and $367 \%$ at $0 \mathrm{~V}$ bias under a flux of $3 \times 10^{4} \mathrm{n} / \mathrm{cm}^{2} / \mathrm{s}$, respectively. An imbalance in the distribution of filled traps between the two electric contacts could explain the self-biased operation of the MSM detectors. Neutron sensitivity is further enhanced with electrical biasing, reaching 316 and $1192 \%$ at $200 \mathrm{~V}$ and a flux of $3 \times 10^{4}$ $\mathrm{n} / \mathrm{cm}^{2} / \mathrm{s}$ for natural and ${ }^{10} \mathrm{~B}$-enriched h-BN detectors, respectively, with dark current as low as $2.5 \mathrm{pA}$ at $200 \mathrm{~V}$. The increased performance under bias has been attributed to a gain mechanism based on neutron-induced charge carrier trapping at the semiconductor/

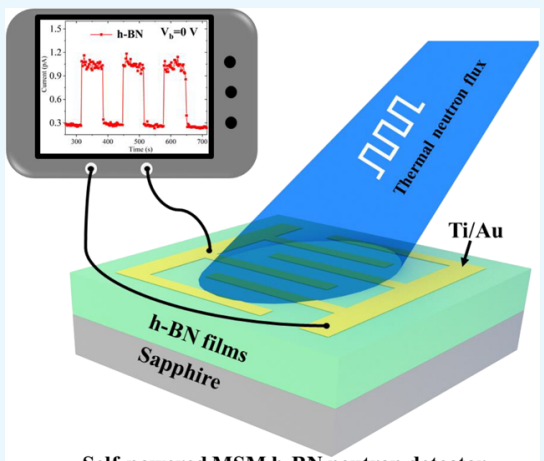
metal interface. The response of the MSM detectors under thermal neutron flux and bias voltages was linear. These results clearly indicate that the thin-film monocrystal BN MSM neutron detectors can be optimized to operate sensitively with the absence of external bias and generate stronger signal detection using ${ }^{10} \mathrm{~B}$-enriched boron.

\section{INTRODUCTION}

Hexagonal boron nitride (h-BN) has extraordinary physical properties such as chemical inertness, high thermal conductivity, strong mechanical strength, and wide band gap ${ }^{1,2}$ making it among the materials of choice for emerging applications in deep UV (DUV) optoelectronics. ${ }^{3,4} \mathrm{~h}-\mathrm{BN}$ is also a uniquely useful material for neutron detection due to its high thermal neutron capture cross section ( 3840 barns for ${ }^{10} \mathrm{~B}$ atoms). In addition, h-BN has weak sensitivity to gamma rays by virtue of its relatively low density $\left(2.2 \mathrm{~g} \mathrm{~cm}^{-3}\right)$, which makes it more attractive for thermal neutron detection than other semiconductor materials. ${ }^{5-7}$ Natural BN contains $20 \%{ }^{10} \mathrm{~B}$ and has an effective absorption length of $277 \mu \mathrm{m}$ for thermal neutrons, which decreases significantly to $47 \mu \mathrm{m}$ if the ${ }^{10} \mathrm{~B}$ is enriched to $100 \%{ }^{8}$ Furthermore, in h-BN-based neutron detectors, neutron capture, carrier generation, and charge collection, all happen in the same layer, making it a good candidate for direct thermal neutron conversion. These advantages can result in h-BN detectors that are smaller and less expensive than no boron-based detectors.

Different types of design and device structures have been used to fabricate solid-state neutron detectors. ${ }^{9-12}$ The metalsemiconductor-metal device (MSM) structures are the simplest to realize because no doping is needed, which is very challenging to achieve for BN materials. ${ }^{13,14}$ MSM designs also result in ultralow dark current, which is highly desirable for large relative sensitivity $\left(\Delta I / I_{\mathrm{d}}\right)$, where $\Delta I$ is defined as the difference between the measured current under irradiation and the dark current $\left(I_{\mathrm{d}}\right)$. Other advantages for MSM structures are the possibility (i) to achieve self-powered operation similar to that demonstrated for UV photodetectors either by using two different metal contacts or the same contacts with specific metal semiconductor interface engineering ${ }^{15-17}$ and (ii) to benefit from internal gain to increase the neutron response signal. ${ }^{18,19}$ McGregor et al. ${ }^{20}$ have demonstrated for the first time the feasibility of pyrolytic BN-based neutron detectors with an efficiency of up to $7.2 \%$ under an electric field of $4 \mathrm{kV}$ / $\mathrm{cm}$. More recently, neutron detectors have been demonstrated with epitaxial monocrystalline h-BN using natural and enriched

Received: October 1, 2021

Accepted: December 14, 2021

Published: December 28, 2021

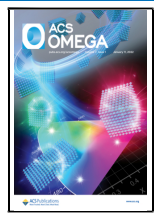




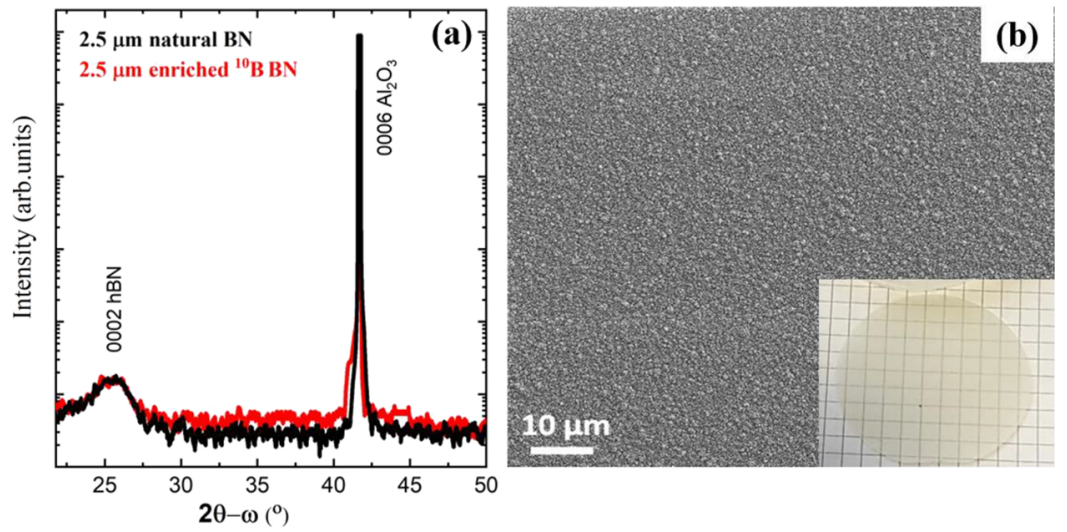

Figure 1. (a) High-resolution X-ray diffraction (HR-XRD) $2 \theta-\omega$ scan of the natural and ${ }^{10} \mathrm{~B}$-enriched BN films. (b) SEM image of $2.5 \mu \mathrm{m}$ thick natural $\mathrm{BN}$ films; the inset shows a photograph of the $2.5 \mu \mathrm{m}$ thick natural $\mathrm{BN}$ films grown on a 2 inch sapphire wafer.

${ }^{10} \mathrm{~B}$ with different thicknesses up to $50 \mu \mathrm{m} .{ }^{21-24}$ These promising results by the group at Texas Tech University (TTU) suggest that more research would be needed to further explore the benefit of h-BN MSM detectors. For instance, the possibility of implementing self-biasing detectors with a gain mechanism has not yet been reported with h-BN. Moreover, since the materials quality of h-BN depends on the layer thickness $^{25,26}$ and growth conditions, ${ }^{25}$ the comparison of the performance of neutron detectors with either a natural or enriched ${ }^{10} \mathrm{~B}$ h-BN active layer would require devices fabricated using the same growth conditions, layer thicknesses, and electric contact fabrication conditions. In this work, we report metal-semiconductor-metal (MSM) neutron detectors based on both $\mathrm{Ti} / \mathrm{Au}$ and $\mathrm{Ni} / \mathrm{Au}$ interdigitated h-BN structures (with natural and ${ }^{10} \mathrm{~B}$-enriched boron) using the same design, and all the devices are fabricated in the same conditions. This study represents a first attempt to evaluate the potential of self-biased operation and gain mechanisms of MSM devices. We have measured the neutron response at zero volts and under strong bias voltage under different thermal neutron fluxes. Even with a relatively thin $\mathrm{BN}$ layer, the MSM detectors based on ${ }^{10} \mathrm{~B}$ enriched h-BN showed four times higher sensitivity when compared to natural h-BN-based detectors.

\section{RESULTS AND DISCUSSION}

Figure 1a shows the $2 \theta-\omega$ scan of the natural and ${ }^{10} \mathrm{~B}$ enriched $\mathrm{BN}$ films, in which a peak located at $25.8^{\circ}$ is related to the (0002) planes of the sp2-BN.

The surface morphology of the $2.5 \mu \mathrm{m}$ thick h-BN layers is shown in Figure 1b. The SEM image reveals a grainy morphology with the absence of wrinkles on the surface. The sample was optically transparent (inset of Figure 1b), and the cross-sectional high-resolution transmission electron microscopy (HRTEM) not shown here revealed the same structural quality as the samples presented in our previous work, ${ }^{25}$ where a thin-layered h-BN structure up to $10 \mathrm{~nm}$ was obtained, with gradual turbostratic stacking appearing gradually as thickness increased. AFM images were used to measure the thickness of the h-BN epi layer. For this, the h-BN layer was partially lifted off from its sapphire substrate (see the Supporting Information). The thickness of the h-BN layer is estimated to be $2.50 \pm 0.07 \mu \mathrm{m}$.

The structural and morphological characteristics of the ${ }^{10} \mathrm{~B}$ enriched h-BN layers are quasi-identical to those of natural h$\mathrm{BN}$ layers. In addition, we were able to lift the ${ }^{10} \mathrm{~B}$-enriched $\mathrm{h}$ -
BN layers off from their sapphire substrates, confirming the presence of a layered h-BN as with natural boron, which is a characteristic of $2 \mathrm{D}$ materials. Based on these results, we can state that the isotope of boron has an impact on neutron capture and not on the structural quality of the h-BN material.

A set of grown $2.5 \mu \mathrm{m}$ natural and ${ }^{10} \mathrm{~B}$-enriched h-BN film samples were then used for fabrication of MSM detectors and were tested under thermal neutron flux.

Figure 2 shows the $I-V$ characteristics of the two MSM $(\mathrm{Ni} / \mathrm{Au}-\mathrm{Ni} / \mathrm{Au})$ and $(\mathrm{Ti} / \mathrm{Au}-\mathrm{Ti} / \mathrm{Au})$ devices for applied

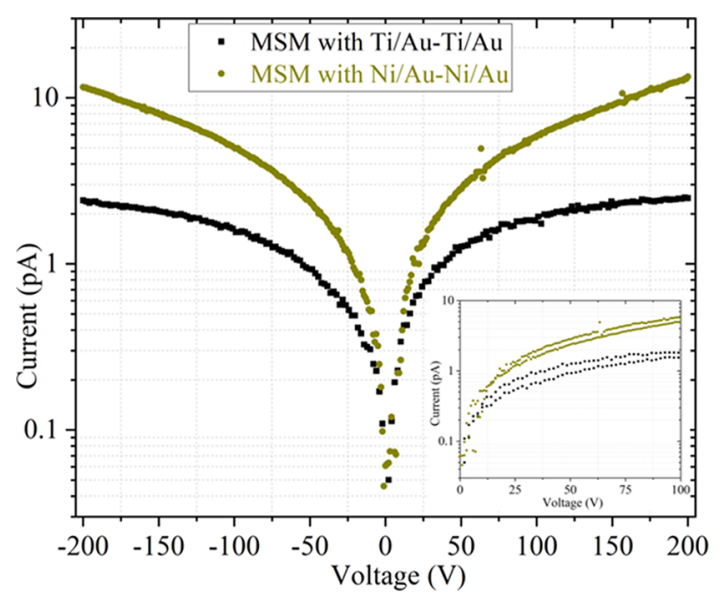

Figure 2. Dark current of the $2.5 \mu \mathrm{m}$ thick natural h-BN MSM detectors with two different Schottky contacts $(\mathrm{Ni} / \mathrm{Au}-\mathrm{Ni} / \mathrm{Au}$ and $\mathrm{Ti} / \mathrm{Au}-\mathrm{Ti} / \mathrm{Au})$. Inset shows the corresponding $I-|V|$ curves to highlight the asymmetric behavior.

voltage in the -200 to $200 \mathrm{~V}$ range recorded in dark condition. Since the work function of $\mathrm{Ti}$ is lower than the work function of $\mathrm{Ni}$, the Schottky barrier height $(\mathrm{SBH})$ formed at the $\mathrm{Ti} / \mathrm{h}$ $\mathrm{BN}$ interface is higher than the one formed using Ni. Thus, as expected, the current flowing in the MSM with Ti contact is lower than the one flowing in the MSM with $\mathrm{Ni}$ contact. Indeed, the current measured at $+/-200 \mathrm{~V}$ in the MSM with $\mathrm{Ni}$ contact is around $12 \mathrm{pA}$, which is five times greater than the current measured in the same condition in the MSM with Ti contact. Even more interestingly, despite the same metal nature of the two contacts, both $I-V$ curves exhibit a slightly nonsymmetric behavior versus the applied voltage as shown in the inset of Figure 2. This asymmetric behavior most likely originates from a different filled carrier trap density at the 

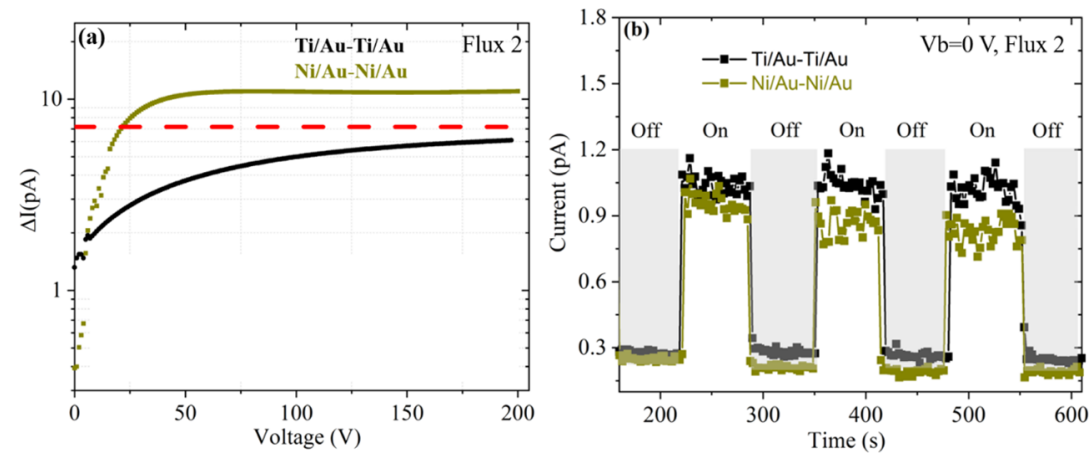

Figure 3. (a) Neutron-induced current of natural h-BN MSM with $\mathrm{Ti} / \mathrm{Au}-\mathrm{Ti} / \mathrm{Au}$ and $\mathrm{Ni} / \mathrm{Au}-\mathrm{Ni} / \mathrm{Au}$ contacts when submitted to a neutron flux of $3 \times 10^{4} \mathrm{n} / \mathrm{cm}^{2} / \mathrm{s}$ (flux 2). (b) Corresponding recording of the current variation at zero bias for both devices.

interface of the h-BN with the two contacts, ${ }^{27}$ leading to different $\mathrm{SBH}$ at the two contacts.

The two types of detectors were then exposed to irradiation with a neutron flow of $3 \times 10^{4} \mathrm{n} / \mathrm{cm}^{2} / \mathrm{s}$. A significant increase of current difference $\Delta I$ versus applied voltage has been observed as shown in Figure 3a.

Since the two MSM devices have asymmetric $\mathrm{SBH}$, a nonzero $\Delta I$ is observed when the devices are unbiased. These zero-bias $\Delta I$ values are probably overestimated because of the noisy signals and low currents. To confirm and refine the value of the unbiased $\Delta I$, we have recorded, as shown in Figure $3 \mathrm{~b}$, the current variation at zero bias for both devices submitted to a periodic neutron flux of $3 \times 10^{4} \mathrm{n} / \mathrm{cm}^{2} / \mathrm{s}$. A clear current variation with a $\Delta I$ of $0.7 \mathrm{pA}$ and sensitivity of $233 \%$, which is similar for both detectors, is obtained.

When the applied voltage is increased, both $\Delta I$ s increase and saturate for voltages above $50 \mathrm{~V}$. At a bias of $200 \mathrm{~V}$ (Figure $3 \mathrm{a})$, the $\Delta I$ of the MSM detector with Ni contact is about twice the one of the MSM detector with Ti contact. Neutron capture by ${ }^{10} \mathrm{~B}$ atoms leads to the generation of daughter particles according to the following equations.

$$
\begin{aligned}
& { }_{5}^{10} \mathrm{~B}+{ }_{0}^{1} n \rightarrow{ }_{3}^{7} \mathrm{Li}+{ }_{2}^{4} \alpha(2.78 \mathrm{MeV})(6 \%) \text {, excited state } \\
& { }_{5}^{10} \mathrm{~B}+{ }_{0}^{1} n \rightarrow{ }_{3}^{7} \mathrm{Li}^{*}+{ }_{2}^{4} \alpha(2.3 \mathrm{MeV})(94 \%) \text {, ground state }
\end{aligned}
$$

The average energy of the daughter particles is $2.34 \mathrm{MeV}$. These charged particles penetrate the BN layers and lose their kinetic energy to create a cloud of electron-hole pairs. The theoretical expected $\Delta I$ corresponding to the collected charge carriers generated by the daughter particles in the case of our detectors is about $7 \mathrm{pA}$ (see the Supporting Information) and is reported on Figure $3 \mathrm{a}$ as the red dotted line. Above $25 \mathrm{~V}$, the $\Delta I$ of the MSM detectors with $\mathrm{Ni}$ is higher than the theoretical value (gain $>1$ ). For MSM detector with $\mathrm{Ti}$, the $\Delta I$ is very close to this value at $200 \mathrm{~V}($ gain $<1)$. This could be explained by a gain mechanism originating from a trapping of the neutron-generated carriers at the metal-semiconductor interfaces, $^{28,29}$ which decreases the SBH, allowing more current to flow. This increase of the current flow can be expressed as

$$
\Delta I^{G}=I_{\mathrm{d}} \exp \left(\frac{\Delta \phi}{k T}\right)-1
$$

where $I_{\mathrm{d}}$ is the dark current, $\Delta \phi$ is the Schottky decrease due to generated carrier trapping, $k$ is the Boltzmann constant, and $T$ is the absolute temperature.
According to eq 3, with the increase of the dark current $\left(I_{\mathrm{d}}\right)$ and decrease of the $\mathrm{SBH}(\Delta \phi)$, the internal gain will increase. If we assume a similar $\Delta \phi$ for both MSM detectors, then devices having the larger dark current should have the largest $\Delta I$, which is the case. Nevertheless, a high gain comes with a large $I_{\mathrm{d}}$, which decreases the sensitivity $\left(\Delta I / I_{\mathrm{d}}\right)$ of the detector. In our case, the MSM detector with Ni contact exhibits a larger dark current and, thus, $\Delta I$ as well as a weaker sensitivity than the MSM detector with Ti contact. For the rest of the study, we have then considered only the high-sensitivity Ti-contact MSM detector.

For the comparison of ${ }^{10} \mathrm{~B}$-enriched and natural detectors, we have used MSM devices with $\mathrm{Ti} / \mathrm{Au}$ metal contacts. Figure $4 \mathrm{a}, \mathrm{b}$ show the zero-voltage neutron-induced current variation
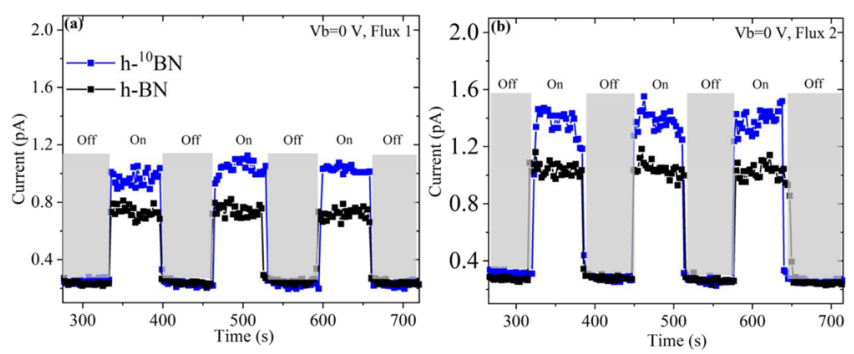

Figure 4. Real-time neutron response of the natural h-BN and ${ }^{10} \mathrm{~B}-$ enriched h-BN MSMs with $\mathrm{Ti} / \mathrm{Au}-\mathrm{Ti} / \mathrm{Au}$ contacts at zero volts with flux 1 (a) and flux 2 (b), respectively.

versus time for devices submitted to $1.5 \times 10^{4} \mathrm{n} / \mathrm{cm}^{2} / \mathrm{s}$ (flux 1) and $3 \times 10^{4} \mathrm{n} / \mathrm{cm}^{2} / \mathrm{s}$ (flux 2). We observed a clear response for both natural and ${ }^{10} \mathrm{~B}$-enriched h-BN MSM devices at zero bias voltage. Higher neutron current of the ${ }^{10} \mathrm{~B}$-enriched MSM device is obtained for both thermal neutron fluxes.

The sensitivities of the natural and ${ }^{10} \mathrm{~B}$-enriched $\mathrm{h}-\mathrm{BN}$ detectors at $0 \mathrm{~V}$ bias are 233 and $367 \%$, respectively. These sensitivities cannot be directly compared with the literature since there is no value reported at zero volts with h-BN, and the sensitivity at applied voltage reported in this material was obtained with other thicknesses. For instance, Li et al. ${ }^{21}$ have reported a current variation of $0.087 \mathrm{pA}$ for a $1 \mu \mathrm{m}$ thick h-BN neutron detector operating under an electric field above $4 \times$ $10^{4} \mathrm{~V} / \mathrm{cm}$, which corresponds to a sensitivity of $170 \%$.

The neutron-induced current at zero volts in the MSM based on natural and ${ }^{10} \mathrm{~B}$-enriched $\mathrm{h}-\mathrm{BN}$ has been compared. The neutron-induced current of ${ }^{10} \mathrm{~B}$-enriched h-BN MSM is about 1.4 times higher compared to the one with natural h-BN MSM at zero voltage (Figure 4). This difference is attributed 

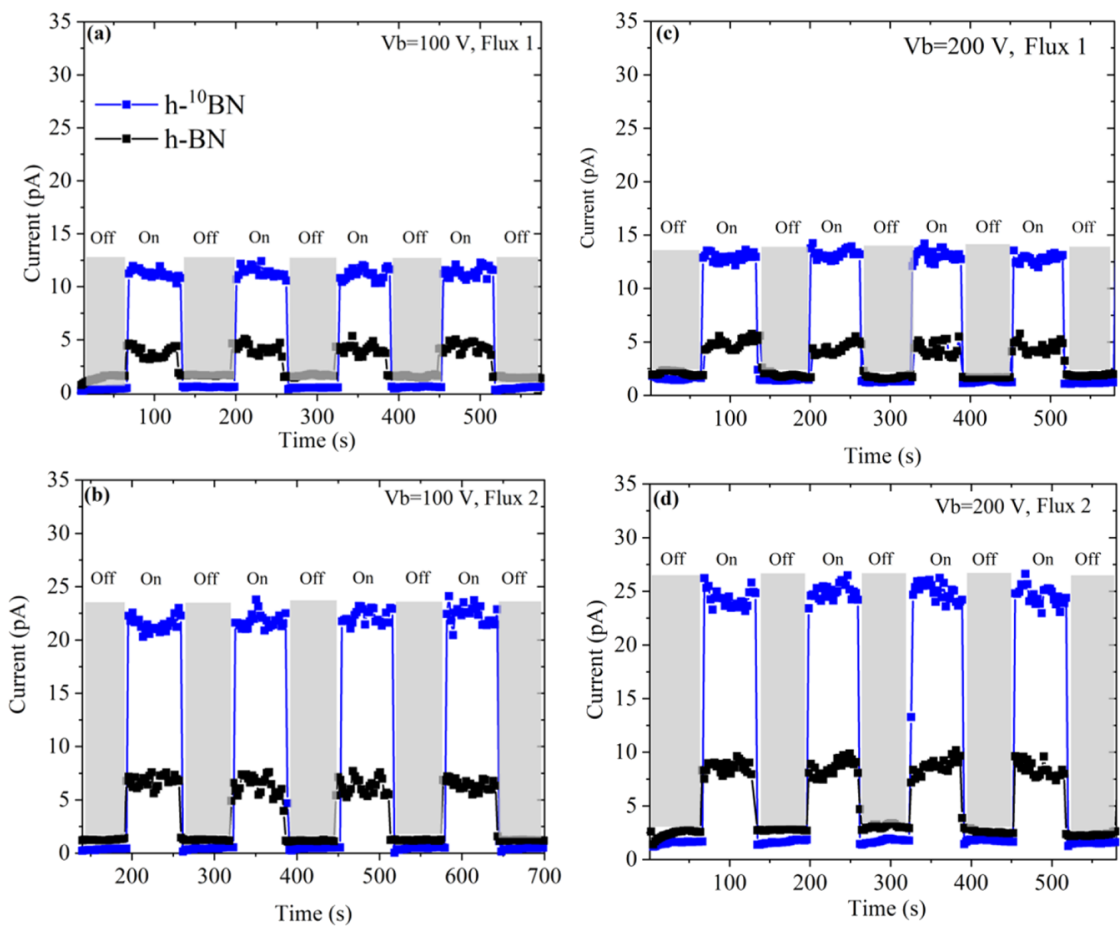

Figure 5. Real-time neutron response of the natural h-BN and ${ }^{10} \mathrm{~B}$-enriched h-BN MSM with $\mathrm{Ti} / \mathrm{Au}-\mathrm{Ti} / \mathrm{Au}$ contacts at 100 and $200 \mathrm{~V}(\mathrm{a}, \mathrm{c})$ under flux 1 and $(b, d)$ under flux 2 .

to the increased ${ }^{10} \mathrm{~B}$ atoms in the enriched h-BN material, resulting in a higher global thermal neutron capture cross section. In that case, more neutrons are absorbed, leading to larger excess free carrier density and, consequently, higher current.

The same detectors (natural and ${ }^{10} \mathrm{~B}$-enriched h-BN MSM with $\mathrm{Ti} / \mathrm{Au}$ ) have been also studied under bias voltages at 100 and $200 \mathrm{~V}$. The obtained results are presented in Figure 5, where the dark current of the two devices is almost the same. This means that the gain mechanism effect will be the same under neutron irradiation. One can observe that the response of the ${ }^{10} \mathrm{~B}$-enriched h-BN MSM is systematically higher than the one of the natural of h-BN for all applied voltages and at different thermal neutron fluxes. Comparing the ${ }^{10} \mathrm{~B}$ effect according to Figure 5, we notice that the ratio of neutroninduced current in ${ }^{10} \mathrm{~B}$ to ${ }^{11} \mathrm{~B}$ films is between 3.2 and 4.5 , which is very close to the theoretically expected value of $5 .^{21}$ By calculating the neutron sensitivity from Figure 5 for flux 2 and under $200 \mathrm{~V}$ bias voltage, it has reached 316 and $1192 \%$ for natural and ${ }^{10} \mathrm{~B}$-enriched $\mathrm{h}-\mathrm{BN}$ detectors, respectively. Since the gain mechanism is present in both devices, we can therefore conclude that this difference is due to ${ }^{10} \mathrm{~B}$-enriched $\mathrm{h}$ BN films. From Figure 5, we notice that the current induced by flux 2 is between 1.78 to $2.38 \pm 0.5$ times the current induced by flux 1 , which shows a good linearity of the detectors. These values are indicative of a remarkable response of the detectors to thermal neutrons.

\section{CONCLUSIONS}

In summary, we used MOVPE to grow $2.5 \mu \mathrm{m}$ natural and ${ }^{10} \mathrm{~B}-$ enriched h-BN layers. At zero bias voltage, a measurable neutron-induced current was detected on h-BN MSM detectors, showing the potential realization of self-powered thermal neutron detectors. The MSM with natural and ${ }^{10} \mathrm{~B}$ enriched h-BN neutron detectors showed a sensitivity to thermal neutrons as high as 233 and $376 \%$ at $0 \mathrm{~V}$, respectively. Linear neutron response of the detectors under thermal neutron fluxes and bias voltages was obtained. In addition, the neutron-induced current of the ${ }^{10} \mathrm{~B}$-enriched h-BN MSM detector is up to four times larger than the one with natural boron. This work shows significant progress for low-cost h-BNbased detection of thermal neutrons.

\section{EXPERIMENTAL SECTION}

Hexagonal boron nitride (h-BN) layers with $2.5 \mu \mathrm{m}$ thickness were grown on 2 inch sapphire substrates by metal-organic vapor phase epitaxy (MOVPE) in an Aixtron close-coupled showerhead (CCS) $3^{\prime \prime} \times 2^{\prime \prime}$ reactor. Triethylboron (TEB) and ammonia $\left(\mathrm{NH}_{3}\right)$ were used as precursors for boron and nitrogen, respectively. For the enriched $\mathrm{BN}$ layers, TEB enriched at $97 \%$ of ${ }^{10} \mathrm{~B}$ isotope has been used. Secondary ion mass spectrometry (SIMS) was used to estimate the ${ }^{10} \mathrm{~B} /{ }^{11} \mathrm{~B}$ content ratio of the $\mathrm{h}-\mathrm{BN}$ layers (see the Supporting Information). The growth was performed at $1280{ }^{\circ} \mathrm{C}$ in hydrogen ambience at $90 \mathrm{mbar}$ and with a growth rate of 470 $\mathrm{nm} / \mathrm{h}$. The layer thickness of $2.5 \mu \mathrm{m}$ has been chosen because above this thickness and with our growth rate $(470 \mathrm{~nm} / \mathrm{h})$, the surface morphology becomes very rough, which can result in poor electrical contacts and poor electrical transport. Because of the very low growth rate, devices that are multiple micrometers thick are impractical for industrial applications, so we concentrate on thicknesses with growth time that remains industrially plausible for MOVPE.

The structural properties were characterized by highresolution X-ray diffraction (HR-XRD) using a Panalytical X'pert Pro MRD system with $\mathrm{Cu} \mathrm{K}_{\alpha}$ radiation in triple axis mode. Detailed structural and optical characterization of 2.5 $\mu \mathrm{m}$ h-BN grown under the same conditions have been performed in our previous work on a UV photodetector. ${ }^{25}$ Morphological properties were characterized using a scanning 
electron microscope (SEM). Electric contacts consist of interdigitate fingers of $200 \mu \mathrm{m}$ width and $200 \mu \mathrm{m}$ spacing. They were fabricated using either a $\mathrm{Ni} / \mathrm{Au}-\mathrm{Ni} / \mathrm{Au}$ bilayer $(30 / 100 \mathrm{~nm})$ or a $\mathrm{Ti} / \mathrm{Au}-\mathrm{Ti} / \mathrm{Au}$ bilayer $(30 / 100 \mathrm{~nm})$ deposited by thermal evaporation through a hard mask. To prevent delamination of the h-BN samples during device fabrication, liquid cleaning was avoided. The surface area of a detector is $1 \times 1 \mathrm{~cm}^{2}$. Figure 6 a shows a picture of the

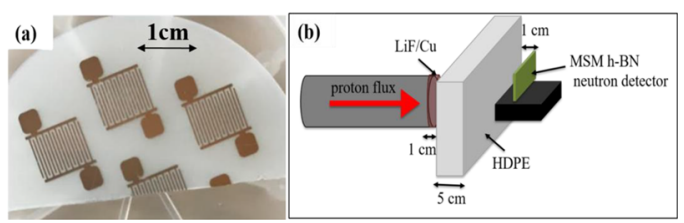

Figure 6. (a) Photograph of the MSM h-BN detectors and (b) schematic of the experimental setup for neutron detection. The $\mathrm{HDPE}$ slab is placed in front of the $\mathrm{LiF} / \mathrm{Cu}$ target, and the distance from the $\mathrm{LiF} / \mathrm{Cu}$ target to the MSM h-BN detector is $7 \mathrm{~cm}$.

fabricated MSM devices. Neutron irradiation of the devices was performed at the Centre Etudes Nucleaires de BordeauxGradignan (CENBG). The neutron source used for this study consists of a $\mathrm{LiF} / \mathrm{Cu}$ target irradiated by a proton beam, which produced $500 \mathrm{keV}$ neutrons. A $5 \mathrm{~cm}$ thick high-density polyethylene (HDPE) slab was inserted between the detector and the target to produce thermal neutrons. As illustrated in Figure $6 \mathrm{~b}$, the HDPE slab was placed $1 \mathrm{~cm}$ away from both the target and the tested devices. The maximum resulting thermal neutron flux was $3 \times 10^{4} \mathrm{n} / \mathrm{cm}^{2} / \mathrm{s}$ with a neutron energy below $1 \mathrm{eV}$. The current-voltage $(I-V)$ of the different devices were recorded at room temperature before, during, and after neutron irradiation using a Keithley $2636 \mathrm{~B}$ source measure unit (SMU).

\section{ASSOCIATED CONTENT}

\section{(s) Supporting Information}

The Supporting Information is available free of charge at https://pubs.acs.org/doi/10.1021/acsomega.1c05458.

h-BN thickness; SIMS data of the ${ }^{10}$ B-enriched h-BN layers; theoretical calculation of the neutron response of the h-BN based detectors; mechanism of the asymmetric Schottky contacts at zero bias (PDF)

\section{AUTHOR INFORMATION}

\section{Corresponding Author}

Abdallah Ougazzaden - CNRS, IRL 2958, GT-CNRS,

57070 Metz, France; School of Electrical and Computer Engineering, GT-Lorraine, Georgia Institute of Technology, 57070 Metz, France; (1) orcid.org/0000-0002-9959-5280; Email: abdallah.ougazzaden@georgiatech-metz.fr

\section{Authors}

Adama Mballo - CNRS, IRL 2958, GT-CNRS, 57070 Metz, France; (1) orcid.org/0000-0001-5778-3702

Ali Ahaitouf - CNRS, IRL 2958, GT-CNRS, 57070 Metz, France; Georgia Tech-Lorraine, 57070 Metz, France

Suresh Sundaram - CNRS, IRL 2958, GT-CNRS, 57070 Metz, France; Georgia Tech-Lorraine, 57070 Metz, France

Ashutosh Srivastava - CNRS, IRL 2958, GT-CNRS, 57070 Metz, France; School of Electrical and Computer Engineering,
GT-Lorraine, Georgia Institute of Technology, 57070 Metz, France

Vishnu Ottapilakkal - CNRS, IRL 2958, GT-CNRS, 57070 Metz, France

Rajat Gujrati - CNRS, IRL 2958, GT-CNRS, 57070 Metz, France; School of Electrical and Computer Engineering, GTLorraine, Georgia Institute of Technology, 57070 Metz, France

Phuong Vuong - CNRS, IRL 2958, GT-CNRS, 57070 Metz, France; 이이. orcid.org/0000-0003-2775-7081

Soufiane Karrakchou - CNRS, IRL 2958, GT-CNRS, 57070 Metz, France; (1) orcid.org/0000-0003-0241-4170

Mritunjay Kumar - Advanced Semiconductor Laboratory, King Abdullah University of Science and Technology (KAUST), Thuwal 23955, Saudi Arabia

Xiaohang Li - Advanced Semiconductor Laboratory, King Abdullah University of Science and Technology (KAUST), Thuwal 23955, Saudi Arabia; 이이이.org/0000-00024434-365X

Yacine Halfaya - Institut Lafayette, 57070 Metz, France Simon Gautier - Institut Lafayette, 57070 Metz, France

Paul L. Voss - CNRS, IRL 2958, GT-CNRS, $57070 \mathrm{Metz}$, France; School of Electrical and Computer Engineering, GTLorraine, Georgia Institute of Technology, 57070 Metz, France

Jean Paul Salvestrini - CNRS, IRL 2958, GT-CNRS, 57070 Metz, France; Georgia Tech-Lorraine, 57070 Metz, France; School of Electrical and Computer Engineering, GT-Lorraine, Georgia Institute of Technology, 57070 Metz, France; (1) orcid.org/0000-0002-0482-1178

Complete contact information is available at:

https://pubs.acs.org/10.1021/acsomega.1c05458

\section{Notes}

The authors declare no competing financial interest.

\section{ACKNOWLEDGMENTS}

This study was partially funded by the French National Research Agency (ANR) under the GANEX Laboratory of Excellence (Labex) project and the KAUST Competitive Research Grant under grant no. OSR-2018-CRG7-3771.2 as well as the Grand Est Region in France. The authors gratefully acknowledge Stephanie Sorieul, Mourad Aiche, and Ludovic Mathieu from CENBG for their help on the neutron testing.

\section{REFERENCES}

(1) Kubota, Y.; Watanabe, K.; Tsuda, O.; Taniguchi, T. Deep Ultraviolet Light-Emitting Hexagonal Boron Nitride Synthesized at Atmospheric Pressure. Science 2007, 317, 932-934.

(2) Watanabe, K.; Taniguchi, T.; Kanda, H. Direct-Bandgap Properties and Evidence for Ultraviolet Lasing of Hexagonal Boron Nitride Single Crystal. Nature Mater 2004, 3, 404-409.

(3) Shim, J.; Bae, S.-H.; Kong, W.; Lee, D.; Qiao, K.; Nezich, D.; Park, Y. J.; Zhao, R.; Sundaram, S.; Li, X.; Yeon, H.; Choi, C.; Kum, H.; Yue, R.; Zhou, G.; Ou, Y.; Lee, K.; Moodera, J.; Zhao, X.; Ahn, J.H.; Hinkle, C.; Ougazzaden, A.; Kim, J. Controlled Crack Propagation for Atomic Precision Handling of Wafer-Scale Two-Dimensional Materials. Science 2018, 362, 665-670.

(4) Geim, A. K.; Grigorieva, I. V. Van Der Waals Heterostructures. Nature 2013, 499, 419-425.

(5) Miyake, A.; Nishioka, T.; Singh, S.; Morii, H.; Mimura, H.; Aoki, T. A CdTe Detector with a Gd Converter for Thermal Neutron Detection. Nucl. Instrum. Methods Phys. Res., Sect. A 2011, 654, 390393. 
(6) Mcgregor, D. S.; Lindsay, J. T.; Olsen, R. W. Thermal Neutron Detection with Cadmium1-x Zincx Telluride Semiconductor Detectors. Nucl. Instrum. Methods Phys. Res., Sect. A 1996, 381, 498-501.

(7) Bell, Z. W.; Pohl, K. R.; van den Berg, L. Neutron Detection with Mercuric Iodide. IEEE Trans. Nucl. Sci. 2004, 51, 1163-1165.

(8) Jiang, H. X.; Lin, J. Y. Review-Hexagonal Boron Nitride Epilayers: Growth, Optical Properties and Device Applications. ECS J. Solid State Sci. Technol. 2017, 6, Q3012-Q3021.

(9) Wielunski, M.; Schütz, R.; Fantuzzi, E.; Pagnamenta, A.; Wahl, W.; Palfálvi, J.; Zombori, P.; Andrasi, A.; Stadtmann, H.; Schmitzer, C. Study of the Sensitivity of Neutron Sensors Consisting of a Converter plus Si Charged-Particle Detector. Nucl. Instrum. Methods Phys. Res. A 2004, 517, 240-253.

(10) Huang, K.-C.; Dahal, R.; Lu, J.-Q.; Danon, Y.; Bhat, I. Boron Filling of Deep Holes for Solid-State Neutron Detector Applications. Trans. Am. Nucl. Soc. 2012, 106, 105-106.

(11) Kitaguchi, H.; Miyai, H.; Izumi, S.; Kaihara, A. Silicon Semiconductor Detectors for Various Nuclear Radiations. IEEE Trans. Nucl. Sci. 1996, 43, 1846-1850.

(12) Polyakov, A. Y.; Smirnov, N. B.; Govorkov, A. V.; Markov, A. V.; Pearton, S. J.; Kolin, N. G.; Merkurisov, D. I.; Boiko, V. M.; Lee, C.-R.; Lee, I.-H. Fast Neutron Irradiation Effects in N-GaN. J. Vac. Sci. Technol., B: Microelectron. Nanometer Struct. - Process., Meas., Phenom 2007, 25, 436-442.

(13) Majety, S.; Doan, T. C.; Li, J.; Lin, J. Y.; Jiang, H. X. Electrical Transport Properties of Si-Doped Hexagonal Boron Nitride Epilayers. AIP Adv. 2013, 3, 122116.

(14) Mballo, A.; Srivastava, A.; Sundaram, S.; Vuong, P.; Karrakchou, S.; Halfaya, Y.; Gautier, S.; Voss, P. L.; Ahaitouf, A.; Salvestrini, J. P.; Ougazzaden, A. Towards P-Type Conduction in Hexagonal Boron Nitride: Doping Study and Electrical Measurements Analysis of $\mathrm{HBN} / \mathrm{AlGaN}$ Heterojunctions. Nanomaterials 2021, 11, 211.

(15) Moon, I. K.; Ki, B.; Yoon, S.; Choi, J.; Oh, J. Lateral Photovoltaic Effect in Flexible Free-Standing Reduced Graphene Oxide Film for Self-Powered Position-Sensitive Detection. Sci. Rep. 2016, 6, 33525.

(16) Huang, C.-Y.; Chen, K.; Chang, C. Realization of a SelfPowered $\mathrm{ZnSnO}$ MSM UV Photodetector That Uses Surface State Controlled Photovoltaic Effect. Ceram. Int. 2021, 47, 1785-1791.

(17) Chen, H.-Y.; Liu, K.-W.; Chen, X.; Zhang, Z.-Z.; Fan, M.-M.; Jiang, M.-M.; Xie, X.-H.; Zhao, H.-F.; Shen, D.-Z. Realization of a Self-Powered ZnO MSM UV Photodetector with High Responsivity Using an Asymmetric Pair of Au Electrodes. J. Mater. Chem. C 2014, 2, 9689-9694.

(18) Salvestrini, J. P.; Ahaitouf, A.; Srour, H.; Gautier, S.; Moudakir, T.; Assouar, B.; Ougazzaden, A. Tuning of Internal Gain, Dark Current and Cutoff Wavelength of UV Photodetectors Using QuasiAlloy of BGaN-GaN and BGaN-AlN Superlattices. In SPIE Photonic West; San Francisco, United States, 2012, 8268, 82682S.

(19) Katz, O.; Garber, V.; Meyler, B.; Bahir, G.; Salzman, J. Gain Mechanism in GaN Schottky Ultraviolet Detectors. Appl. Phys. Lett. 2001, 79, 1417-1419.

(20) McGregor, D. S.; Unruh, T. C.; McNeil, W. J. Thermal Neutron Detection with Pyrolytic Boron Nitride. Nucl. Instrum. Methods Phys. Res. Sect. A 2008, 591, 530-533.

(21) Li, J.; Dahal, R.; Majety, S.; Lin, J.-Y.; Jiang, H. X. Hexagonal Boron Nitride Epitaxial Layers as Neutron Detector Materials. Nucl. Instrum. Methods Phys. Res. Sect A 2011, 654, 417-420.

(22) Doan, T. C.; Majety, S.; Grenadier, S.; Li, J.; Lin, J. Y.; Jiang, H. X. Hexagonal Boron Nitride Thin Film Thermal Neutron Detectors with High Energy Resolution of the Reaction Products. Nucl. Instrum. Methods Phys. Res. Sect A 2015, 783, 121-127.

(23) Doan, T. C. Thermal Neutron Detector Based on Hexagonal Boron Nitride; $\mathrm{PhD}$ diss Texas Tech University Libraries: 2016, 150.

(24) Maity, A.; Grenadier, S. J.; Li, J.; Lin, J. Y.; Jiang, H. X. Hexagonal Boron Nitride Neutron Detectors with High Detection Efficiencies. J. Appl. Phys. 2018, 123, No. 044501.
(25) Li, X.; Jordan, M. B.; Ayari, T.; Sundaram, S.; El Gmili, Y.; Alam, S.; Alam, M.; Patriarche, G.; Voss, P. L.; Salvestrini, J. P.; Ougazzaden, A. Flexible Metal-Semiconductor-Metal Device Prototype on Wafer-Scale Thick Boron Nitride Layers Grown by MOVPE. Sci. Rep. 2017, 7, 786.

(26) Li, X.; Sundaram, S.; El Gmili, Y.; Ayari, T.; Puybaret, R.; Patriarche, G.; Voss, P. L.; Salvestrini, J. P.; Ougazzaden, A. LargeArea Two-Dimensional Layered Hexagonal Boron Nitride Grown on Sapphire by Metalorganic Vapor Phase Epitaxy. Cryst. Growth Des. 2016, 16, 3409-3415.

(27) Wang, H.-C.; Hong, Y.; Chen, Z.; Lao, C.; Lu, Y.; Yang, Z.; Zhu, Y.; Liu, X. ZnO UV Photodetectors Modified by Ag Nanoparticles Using All-Inkjet-Printing. Nanoscale Res. Lett. 2020, 15,176

(28) Sang, L.; Liao, M.; Koide, Y.; Sumiya, M. High-Temperature Ultraviolet Detection Based on InGaN Schottky Photodiodes. Appl. Phys. Lett. 2011, 99, No. 031115.

(29) Zhang, W. J.; Kanda, H.; Matsumoto, S. Cathodoluminescence of Cubic Boron Nitride Films Deposited by Chemical Vapor Deposition. Appl. Phys. Lett. 2002, 81, 3356-3358.

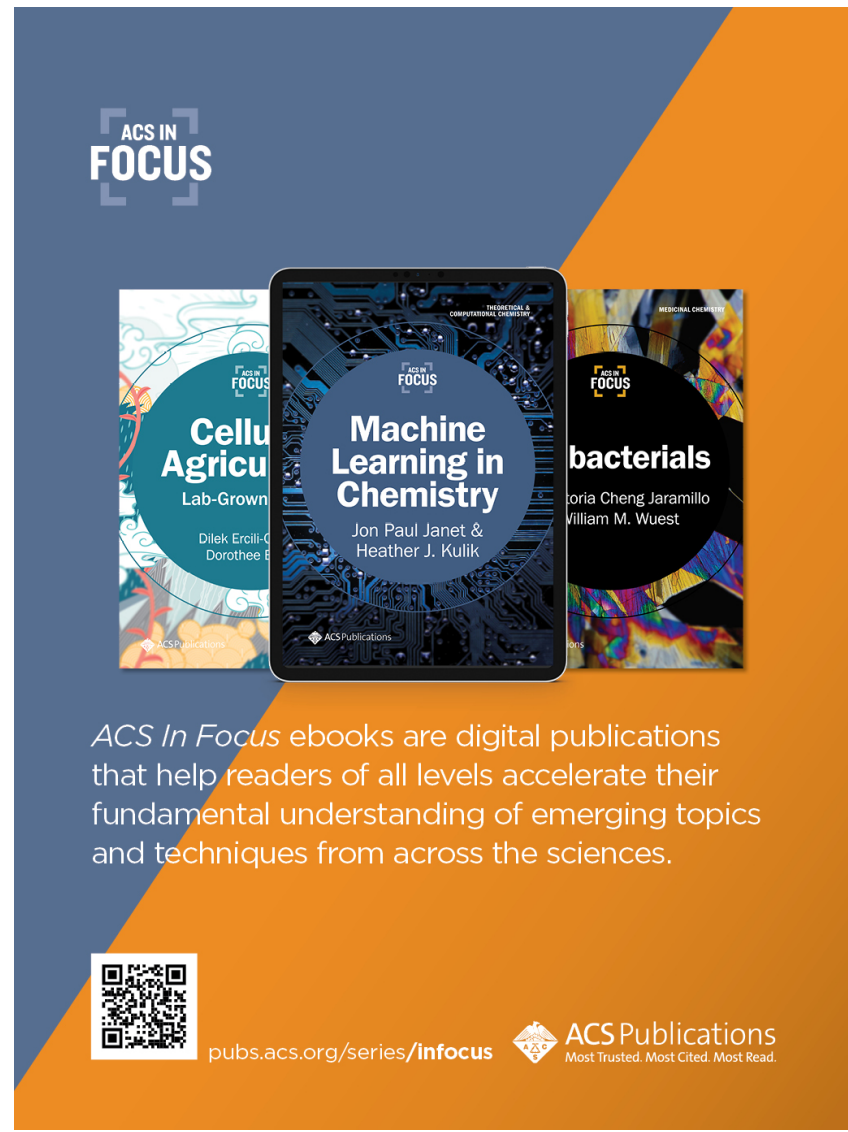

